\title{
Outcomes and Safety Issues Related to Percutaneous Endoscopic Gastrostomy in Neurodegenerative Diseases
}

\author{
Yun Jeong Lim \\ Department of Internal Medicine, Dongguk University, College of Medicine, Dongguk University Ilsan Hospital, Goyang, Korea
}

See "Percutaneous Endoscopic Gastrostomy Tube Insertion in Neurodegenerative Disease: A Retrospective Study and Literature Review" by Pamela Sarkar, Alice Cole, Neil J. Scolding, et al., on page 270-278.

Enteral nutrition via gastric tube insertion with an endoscopic guide is a very efficient method for patients who are unable to swallow food. ${ }^{1}$ The use of percutaneous endoscopic gastrostomy (PEG) has been found to be useful in many situations. Common indications for PEG are neurological disorders (dysphagia after stroke, multiple sclerosis, Guillain-Barré syndrome, Parkinson's disease, cranial trauma, amyotrophic lateral sclerosis, poliomyelitis, and others), oncologic disorders (such as head and neck cancer), and other clinical conditions that result in impaired or diminished swallowing ability, although the patients have a functional digestive system. ${ }^{1,2}$ However, the decision about whether to use PEG tube placement should be individualized to minimize complications. Identifying risk factors for PEG tube insertion and an early decision regarding the use of gastric tube system for feeding are very important for achieving marked improvement in a patient's nutritional status, general well-being, and prognosis.

Early major PEG procedure-related complications include gastrostomy puncture-related hemorrhage, peritonitis, wound infections, injury to adjacent organs (particularly the colon),

\section{Received: March 28, 2017 Accepted: April 6, 2017}

Correspondence: Yun Jeong Lim

Department of Internal Medicine, Dongguk University, College of Medicine, Dongguk University Ilsan Hospital, 27 Dongguk-ro, Ilsandong-gu, Goyang 10326, Korea

Tel: +82-31-961-7133, Fax: +82-31-961-7730, E-mail: drlimyj@gmail.com

(c) This is an Open Access article distributed under the terms of the Creative Commons Attribution Non-Commercial License (http://creativecommons.org/ licenses/by-nc/3.0) which permits unrestricted non-commercial use, distribution, and reproduction in any medium, provided the original work is properly cited. and aspiration. ${ }^{3}$ To avoid these early complications, first, positioning a safe gastric puncture point is very important. ${ }^{4} \mathrm{~A}$ colo-cutaneous fistula is a rare complication, and it occurs when the colon is accidentally punctured during guided needle puncture for initial gastrostomy placement. It seldom occurs due to internal bump-induced erosion into the adjacent colon over time. ${ }^{3,5}$ Second, the entire procedure should be performed under aseptic conditions. A skin incision of the proper length should be made on the abdominal wall with a knife for easy passage of the gastric tube into the stomach cavity. Aspiration can be prevented by frequent intra-procedural suction of the oral cavity, placing the patient in the left lateral position, and avoiding excessive air insufflation. The tube site should be kept clean, and the external bolster should be carefully secured to avoid inadvertent tube removal. ${ }^{2,5}$ The stoma tract usually matures within 7-14 days after initial gastrostomy. ${ }^{2,5}$ To avoid inadvertent removal of the gastric tube, physicians should be particularly careful in patients with arm movement and mental confusion. Fluids and medications are usually started 4 hours after the procedure. ${ }^{2}$ Although early feeding does not increase the risk of complications, feeding can be delayed due to the fear of wound infection. Daily review of the stoma site following PEG tube insertion should be performed. Positioning the external bolster approximately $1-2 \mathrm{~cm}$ from the abdominal wall is important for minimizing late complications, such as internal bolster-induced gastric ulcers, granulomas (proliferation of granulation tissue through the stoma), and others. ${ }^{3}$ Generally, loose positioning is recommended because it prevents pressure necrosis and buried bumper syndrome. 
However, tight apposition of the external bolster for at least the first 4 days after the procedure can be helpful in preventing gastric leakage. ${ }^{3}$

Neurodegenerative diseases present challenges to feeding and nutrition, but they have not been previously extensively studied despite increasingly widespread recommendations for PEG. Moreover, patients who have neurological diseases with an altered mental status do not have the ability to sense their symptoms and communicate about the procedure. Concerns regarding the relatively high complication rate after PEG tube placement have been raised in patients with neurodegenerative diseases. ${ }^{6,7}$ In this issue of Clinical Endoscopy, Sarkar et al. provide valuable information regarding the safety and utility of PEG in patients with neurodegenerative diseases. ${ }^{8}$ The high rate of complications is likely due to the inherent morbidity and poor condition associated with neurodegenerative diseases, poor baseline nutritional status, risk factors for aspiration associated with neurodegenerative diseases, and long in-patient stay with consequently increased susceptibility to infection. ${ }^{8}$ Therefore, earlier consideration of PEG is helpful under close monitoring of nutrition markers, such as a low albumin level. ${ }^{8,9}$ Age greater than 75 years has been reported to be associated with poor outcomes. ${ }^{8,10,11}$ The European Society for Clinical Nutrition and Metabolism (ESPEN) guidelines recommend that PEG tube placement should be considered prior to the forced vital capacity falling below $50 \% .{ }^{1}$ Careful patient selection, optimal timing of PEG tube insertion, and peri-procedural care with comprehensive education of patients and care-givers, as well as prompt hospital discharge, should be considered to reduce the relatively high morbidity and mortality of PEG in patients with neurodegenerative diseases. ${ }^{8}$
Conflicts of Interest

The author has no financial conflicts of interest.

\section{REFERENCES}

1. Löser C, Aschl G, Hébuterne X, et al. ESPEN guidelines on artificial enteral nutrition--percutaneous endoscopic gastrostomy (PEG). Clin Nutr 2005;24:848-861.

2. Friginal-Ruiz AB, Lucendo AJ. Percutaneous endoscopic gastrostomy: a practical overview on its indications, placement conditions, management, and nursing care. Gastroenterol Nurs 2015;38:354-366; quiz 367368.

3. Hucl T, Spicak J. Complications of percutaneous endoscopic gastrostomy. Best Pract Res Clin Gastroenterol 2016;30:769-781.

4. Chang WK, Hsieh TY. Safety of percutaneous endoscopic gastrostomy in high-risk patients. J Gastroenterol Hepatol 2013;28 Suppl 4:118-122.

5. Stayner JL, Bhatnagar A, McGinn AN, Fang JC. Feeding tube placement: errors and complications. Nutr Clin Pract 2012;27:738-748.

6. Sanders DS, Carter MJ, D'Silva J, James G, Bolton RP, Bardhan KD. Survival analysis in percutaneous endoscopic gastrostomy feeding: a worse outcome in patients with dementia. Am J Gastroenterol 2000;95:14721475.

7. Chiò A, Finocchiaro E, Meineri P, Bottacchi E, Schiffer D. Safety and factors related to survival after percutaneous endoscopic gastrostomy in ALS. ALS percutaneous endoscopic gastrostomy study group. Neurology 1999;53:1123-1125.

8. Sarkar P, Cole A, Scolding NJ, Rice CM. Percutaneous endoscopic gastrostomy tube insertion in neurodegenerative disease: a retrospective study and literature review. Clin Endosc 2017;50:270-278.

9. Tominaga N, Shimoda R, Iwakiri R, et al. Low serum albumin level is risk factor for patients with percutaneous endoscopic gastrostomy. Intern Med 2010;49:2283-2288.

10. Arora G, Rockey D, Gupta S. High In-hospital mortality after percutaneous endoscopic gastrostomy: results of a nationwide population-based study. Clin Gastroenterol Hepatol 2013;11:1437-1444.e3.

11. Janes SE, Price CS, Khan S. Percutaneous endoscopic gastrostomy: 30day mortality trends and risk factors. J Postgrad Med 2005;51:23-28; discussion 28-29. 